\title{
An energy Conservation Scheme in Video Monitoring Storage Systems
}

\author{
Haifeng Zhong, Min Tu \\ Dept. of Safety Management, Jiangxi police college \\ Email: bill_zhf@sina.com
}

Keywords: Streaming media storage, Data layout, Disk system

\begin{abstract}
Disk-based network video monitoring storage system has become a popular trend. By careful analysis on video monitoring access characteristics, this paper proposed a low-power hard disk storage scheme which design a specialized data layout methods and disk access model to improve the capabilities of video storage server to support concurrent video data streaming. By means of reducing the number of disks working in high energy consumption, the scheme ultimately achieving the purpose of saving the energy consumption of the storage system.
\end{abstract}

\section{Introduction}

Digital high-definition network video surveillance system has brought the explosive growth of digital video data. Thus hard disk has become the main video monitor recording equipment because of its unique high-capacity, high performance and low price advantage[1]. At present, the large capacity storage system based on hard disk has become a core component of video surveillance system. Take the video surveillance system using D1 format image as an example, the data rate of each $\mathrm{D} 1$ channel is $2 \mathrm{Mb} / \mathrm{s}[2]$. And the system usually works $7 * 24$ hours. So the one-day video recording takes about 50G storage space. The surveillance system with 500 cameras produces a combined stream of more than 100MB / s, which reaching the average read and write speed of large data on a single hard disk. And the increasingly popular H.264 protocol used high-definition video signal stream over 6Mbps will bring the storage system greater pressure[3] .

Video surveillance system storage system must have both very high capacity and I/O performance. However, a growing number of disk storage system also bring the storage server's high energy consumption and heat dissipation problems[4]. So this paper proposes a energy Conservation Video monitoring file system(ECVMF). According to the characteristics of video monitoring data stream and the disk energy consumption, ECVMF design a special data layout methods to realize the high $\mathrm{I} / \mathrm{O}$ performance and low energy consumption of video surveillance storage system.

\section{Related works}

Video monitoring system is a typical I/O intensive applications. Because the system often contains hundreds of cameras, works continuously and produces constant bit rate, the disk storage systems usually need to support multiple concurrent flow. As a result, disk I/O performance become a major bottleneck for the system. Therefore, The system urgently needs to improve the ability to read and write large chunks of data concurrently. In addition, due to the limited capacity of the system the video data stored in each disk of the system has a certain life cycle. That is say, it is necessary to continuously overwrite the old data with new video data. For many existing file systems, after many such coverage, a large number of disk fragments will inevitably be generated and I/O performance 
will be reduced.

Many research design the specialized file system for controlling the layout of data on disk[5], reducing file fragments, increasing the sequential access rate so as to improve the energy consumption of the disk[6]. In addition, many energy saving technologies focus on dynamic reduction of the number of high energy disks[7], aggregation of hotspot data on some disks and dynamic configuration of cache devices[8].

\section{System structure}

The energy-saving video surveillance storage server based on ECVMF unifies the network data storage service of the network camera to the specialized storage server so that the video data stream distributed on the network can be stored collectively. The model of ECVMF server is shown in Figure 1.

The system uses a hierarchical software structure. And the application service module manages and dispatches requests for data storage from each camera. When the camera produces a video stream data, it issues I / O request to the ECVMF. According to the feedback information from database service module and file service module the application service module determines the specific physical location in disk where the video data stream is written in. Then the video stream is directly transferred to the driver to write in the specified hard disk. And then the metadata database of the video clip is modified. Through the above scheme the ECVMF file system and the metadata database together implement the efficient disk access of the video stream data.

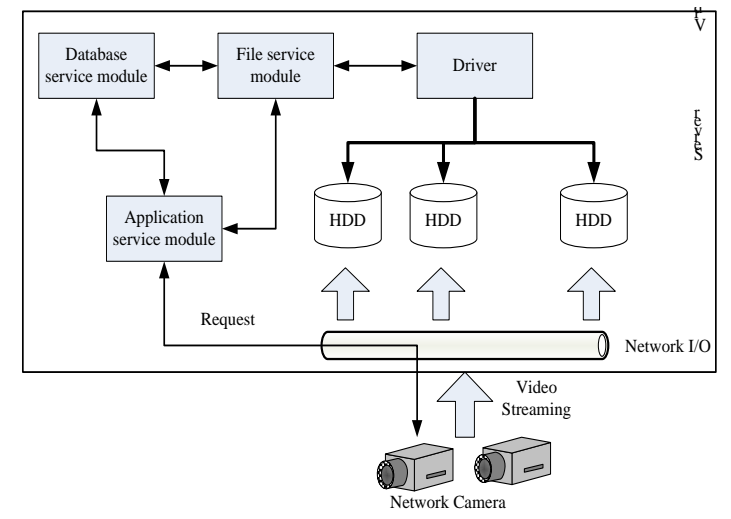

Figure 1. Video Monitoring Server Model

\section{Disk space management}

Let $\mathrm{T}_{\mathrm{IO}}$ be the disk access time to which the system completed a data read or write. Let $\mathrm{T}_{\mathrm{s}}$ be the time to which the head moved radially to the physical track. Let $T_{r}$ be the time to which the head rotates to the target sector. And let $\mathrm{T}_{\mathrm{rw}}$ be the head read / write time. Then $\mathrm{T}_{\mathrm{IO}}$ is given by $\mathrm{T}_{\mathrm{IO}}=\mathrm{T}_{\mathrm{s}}+\mathrm{T}_{\mathrm{r}}+\mathrm{T}_{\mathrm{rW}}$

From the above equation, disk access performance is directly subject to the head positioning delay time Ts+Tr. If the head move shorter distance and the disk rotate less time the files will require less access time. because the data read and write to physically adjacent disk blocks the disk avoid the frequent head moving and therefore improve the efficiency of its access, so the sequential access mode is an effective method to improve the performance of file storage.

File storage mode of traditional video surveillance system separates the relationship between the logic blocks and the corresponding file disk physical blocks. when the video concurrent write disk, video data is written into disk under the state of disorder, thus result in low system performance. In order to realize the video data recording disk sequential access, ECVMF system uses the SQLserver database to establish the mapping relation between the corresponding file logic 
disk blocks and physical location, as shown in table 1 .

Table 1. File Mapping Table

\begin{tabular}{|l|l|l|l|l|l|l|l|}
\hline $\begin{array}{l}\text { Seq. } \\
\text { ID }\end{array}$ & state & $\begin{array}{l}\text { Disk } \\
\text { ID }\end{array}$ & LSB & $\begin{array}{l}\text { Physical } \\
\text { sector }\end{array}$ & Creat time & $\begin{array}{l}\text { Camera } \\
\text { ID }\end{array}$ & $\begin{array}{l}\text { File } \\
\text { ID }\end{array}$ \\
\hline 1 & & 15 & 1 & $1 / 2 / 3 / 4$ & 1702012302 & & A \\
\hline$\ldots$ & $\ldots$ & $\ldots$ & & $\ldots$ & $\ldots$ & $\ldots$ & $\ldots$ \\
\hline 150 & & 2 & 18 & $1 / 2 / 3 / 4$ & 1702051105 & & D \\
\hline$\ldots$ & $\ldots$ & $\ldots$ & & $\ldots$ & $\ldots$ & $\ldots$ & $\ldots$ \\
\hline
\end{tabular}

Table 1 records the creation time, status, corresponding physical device and its disk location information, the block number in the system logical volume, and so on. And disk block are sorted by their physical location and arranged in a monotonically increasing relationship.

Nowadays, many commercial hard drive products such as Hitachi's Deskstar 7k 3000 have three working states: standby, idle and active. In the standby state the hard disk stops rotating. At this time the hard disk consumes the least energy, about $1 \mathrm{w}$. In the active state hard disk response data read and write requests. It consumes the most energy, about $10 \mathrm{w}$. In the idle state the hard disk is rotating and waiting for data read and write requests. It consumes energy in the middle, about $6 \mathrm{w}$. This will meet the high quality video I / O load quality of service under the premise of as much as possible to improve the data throughput of a single disk, making data access focused on a small number of hard drives, thereby reducing the active hard disk to reduce the system's energy consumption.

\section{Energy saving disk scheduling algorithm}

Special disk scheduling algorithm is designed in ECVMF system which merging concurrent multi-channel video data streaming. It uses logical block as the basic unit. And each logical block can save not only fixed video frame data, but also the next writable logic block address associated with the front and back logical block. Due to the large amount of data per video stream, each video record file needs to occupy $\mathrm{n}$ logical disk blocks and use the large data volume writing mode. that is, when the data stream reaches the $\mathrm{N}$ logic block It only write disk once . The data configuration of its storage unit is shown in figure 2. Camera B produces continuous data streams such as video frames F1, F2, and F3. Several frames are encapsulated into a logical disk block such as B1, B1, B2, and B3, which are the continuous data blocks stored in the disk HDD 1 by the camera B.

Before the first use of the ECVMF system, it need to initialize and create logical block in accordance with the specified size. And then create a database file to maintain map information between logic blocks and physical blocks, and describe the logic block allocation information file, including video file name, time of creation and logic block allocation status information and so on. In the process of data storage, the ECVMF system first queries the database table, gets an empty block of logic from the table, and then begins writing operations. If the storage space is full, ECVMF will cover the earliest video files with the latest video stream. In the running process of the system, ECVMF continuously according to combining data stream and the number of disk data flow rate calculation with port number. 


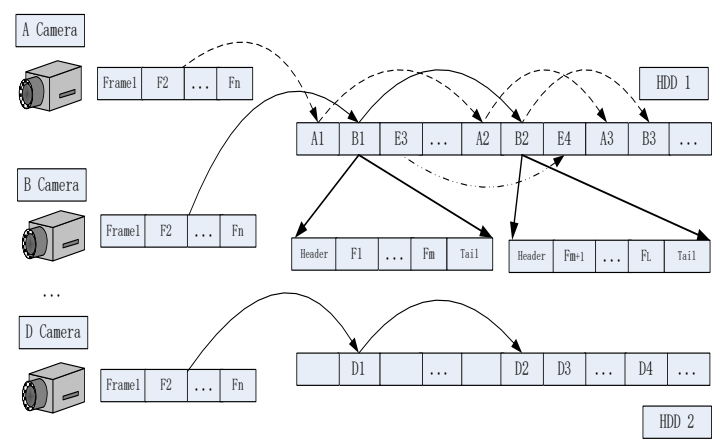

Figure 2. Video File Block Structure

Assuming that the system consists of $i$ disks and each disk can support a load of $C_{i}$. Let $j$ be the number of data stream channel, let $S_{j}$ be data rate of each channel. Then the total load demand of the system $\mathrm{L}_{\mathrm{i}}$ is given by

$$
\mathrm{L}=\sum_{\mathrm{j}=1}^{\mathrm{n}} \mathrm{Sj}
$$

In order to meet the system's storage load, the least number of disks in storage server keeping active state is given by

$$
A=\left[\begin{array}{lll}
L_{i} / C_{i}
\end{array}\right]
$$

ECVMF uses the disk scheduling algorithm which merge multi-channel video data flow. It places data block as much as possible to make the active hard disk full load working state of writing. So reduces the number of the hard disk in active state. The following algorithm determines the storage location of current new video data stream.

a) The new video stream stores $S_{j+1}$ request and gets a response.

b) If $\left[\mathrm{L}_{\mathrm{i}}+\mathrm{S}_{\mathrm{j}+1} / \mathrm{C}_{\mathrm{i}}\right]>\mathrm{A}$, Select $\mathrm{HD}_{\mathrm{j}}$ from the hard disk array in the idle state as the storage hard disk of the data stream.

c) Otherwise, select the active hard disk $\mathrm{HD}_{\mathrm{j}}$ without full load working as the storage hard disk of the data stream.

d) When a request to cancel the video stream $S_{j}$ is received.

e) If $\left[\mathrm{L}_{\mathrm{i}}-\mathrm{S}_{\mathrm{j}} / \mathrm{C}_{\mathrm{i}}\right]<\mathrm{A}$, The hard disk $\mathrm{HD}_{\mathrm{j}}$ storing the video stream $\mathrm{S}_{\mathrm{j}}$ is changed from the active state to the idle state or the standby state according to the system load.

f) Otherwise, cancel the storage request for the video data stream $S_{j}$ in the hard disk HDj.

The system will periodically check the working status of the disk to ensure timely response to the new video data storage requests.

\section{Simulation Energy comparison}

In order to evaluate the effect of the scheme on energy saving, the simulation environment is used and the assumed simulation setting is shown in Table 1. The simulation setting contains 50 virtual hard disks, each disk with a large data average write speed of 120MB / s. We assume that the energy consumption of the hard drive in three working states are $10 \mathrm{w}$ for active, $6 \mathrm{w}$ for idle and $1 \mathrm{w}$ for standby respectively. To simulate video data we use D1 format $720 \mathrm{P}$ video stream with rate of $2 \mathrm{Mb}$ / s. 
Table 2. Simulation settings

\begin{tabular}{|l|l|}
\hline Parameter & Value \\
\hline Disk write performance & $120 \mathrm{MB} / \mathrm{sec}$ \\
\hline Number of virtual hard drives & 50 \\
\hline Energy consumption in active & $10 \mathrm{w}$ \\
\hline Energy consumption in idle & $6 \mathrm{w}$ \\
\hline Energy consumption in standby & $1 \mathrm{w}$ \\
\hline The data rate of the video & $2 \mathrm{Mb} / \mathrm{sec}$ \\
\hline
\end{tabular}

In simulation, we compare the energy consumption of the ECVMF mode and the randomly selection mode. The randomly mode determine the hard disk location to store the data block randomly regardless of energy saving. we change the request number of data channels varies from 100 to 1600 to compare the energy consumption of both schemes. Each data channel contains 10 D1 format camera.

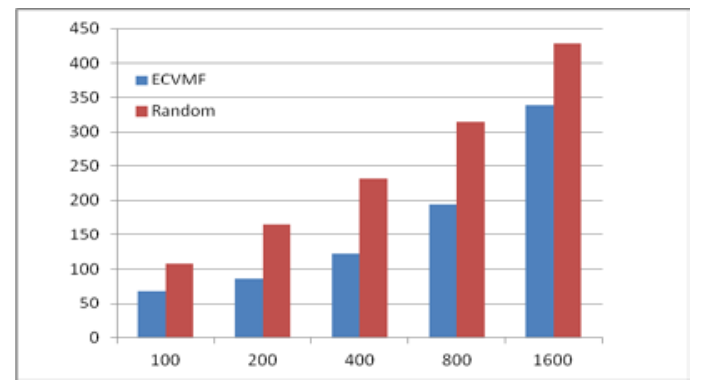

Figure 3. Simulation results of energy consumption

Figure 3 depicts the energy consumption of the two schemes under different load conditions. The vertical axis describes the relative energy consumption of both schemes. The results show that compared with the randomly selection scheme, the proposed scheme reduces the energy consumption under various load conditions. And under low load and full load conditions, the energy consumption reduction ratio is small, while in the medium load conditions, energy consumption is more obvious. Due to under the condition of light load and full load, the disk ratio in 3 states between two schemes is close, but the proportion under the medium load case is quite different.

\section{Conclusion}

Modern video surveillance system is based on video storage data technology. For the large-scale high-definition monitoring data storage system, in addition to requiring high concurrency processing performance it is also essential requirement to achieve environmental protection and energy saving. Aiming at the defects of separating the relationship between file data storage and its disk physical blocks in traditional streaming media storage system, in the paper we design a video data stream storage scheme with fixed size file and new scheduling algorithm to overcome file blocks fragmentation problems. In the scheme the database technology is also used to achieve the centralized management of logic file block and its physical disk block. The simulation results show that the ECVMF model can reduce the energy consumption of the storage system by improving the performance of supporting concurrent data streams, which provides a guarantee for the security and reliable storage of the video data, compared with the random access mode of the traditional streaming media storage system.

\section{Acknowledgements}

This work is supported by Science and Technology Project Founded by the Education Department 
of Jiangxi Province under Grant No.14771 and No.12765

This work is supported by Project Study on the Model of Forensics in Cloud Storage Environment Founded by the Jiangxi Police College

\section{Reference}

[1] G..Gibson et al. "File Server Scaling with Network-Attached Secure Disks”. ACM SIGMETRICS, June 1997, pp.3-5.

[2] M.Greiffenhagen, D.Comaniciu, H.Niemann, et al. “Analysis and Engineering of Vedio Monitoring Systems:An Approach and a Case Study”. Proceeding of the IEEE, 2001, 90(10):

[3] H. Schwarz, D. Marpe, and T. Wiegand, "Overview of the scalable video coding extension of the H. 264/AVC standard," IEEE Trans. Circuits Syst. Video Techn., vol. 17, no. 9, pp.1103-1120,

[4] D. Forte and A. Srivastava. "Energy-aware video storage and retrieval in server environments". In Proceedings of the IEEE International Conference on Green Computing, 2011.pp. 1-6,

[5] H. Amur, J. Cipar, and V. Gupta, "Robust and Flexible Power-Proportional Storage," Proc. First ACM Symp on Cloud Computing, June 2010, pp.51-59

[6] Yi-Cheng Tu and Xiaorui Wang. “A System for Energy-Efficient Data Management”. ACM SIGMOD Record 43(1): May 2014, pp.21-26

[7] . E. Pinheiro and R. Bianchini. "Energy conservation techniques for disk-array-based servers”. In Proceedings of the ACM/IEEE Conference on Supercomputing, June 2004, pp.88-95.

[8] M. Song. "Dynamic buffer allocation for conserving disk energy in clustered video servers which use replication”. In Proceedings of the Embedded and Ubiquitous Computing (EUC) Conference, August 2006, pp.193-203 